\title{
Septicemia por gérmenes anaerobios en lactantes***
}

\author{
Drs. Alfredo Misraji, ** Lionel Bemier," T.M. Ema del Canto, ${ }^{* *}$ Rosalba Urbina, ** E.U. Estela Domic*
}

\begin{abstract}
50 petients under 2 years of age, bospitalized in the Infant Unit of the Roberto del Rio Hospital were studied prospectively during May 1979 to July 1980 . The patients were admitted with different diagnosis and presented at admission or during their stay in the hospital signs of severe infection.

Serial blood cultures were practiced in search of aerobic and anaerobic bacteria. Anaerobic micro-organisms were isolated in six patients, 3 of them had only clinically significant bacteremia according to our criteria. The etiological agents identified were Bacteroides fragilis, Clostridium perfringens and Peptacoccus sacharolyticus in association with Klebsiella pneumoniae.

This experience illustrates the importance of the search for anaerobic bacteria in cases of small children with severe infectious diseases.
\end{abstract}

Los primeros hallazgos relacionados con la presencia de microorganismos anaerobios se deben al químico francés Pasteur, quien al estudiar el proceso de fermentación butírica observó que ésta ocurria en ausencia de oxígeno.

En 1889, Kitasato logró por primera vez cultivos puros de Clostridium tetani, en medios sólidos. En 1893, Veillón aisló Micrococcus anaerobio obligado a partir de una muestra de pus en una Bartolinitis.

Años más tarde este mismo autor y Zuber describieron la presencia de bacterias anaerobias en 25 casos de gangrena supurativa.

El azote de la gangrena en las 2 últimas guerras mundiales, las infecciones por tétano y botulismo, llevaron a numerosos investigadores a estudiar las características biológicas y patogénicas de los microorganismos anaerobios.

Posteriormente, las investigaciones se han dirigido a establecer el rol que estos microorganismos pudieran tener en la etiologia de infecciones graves septicémicas de adultos y niños.

A este respecto, Wilson, Jeffery y colaboradores, en 1971, hicieron una investigación a 264 pacientes con hemocultivos positivos por gérmenes anaerobios. En ella se logró establecer que sólo un $25 \%$ de estas bacteremias eran clínicamente significativas (fiebre, taquicardia, leucocitosis, shock, hipotension, oliguria).

Los gérmenes aislados con mayor frecuencia por estos autores, en los procesos de bacteremias

\footnotetext{
-Hospital Roberto del Rro.

- Departamento de Microbiologa y Parasitologia, Faculend de Medicina Norte.

***Este trabajo fue financiado por Labonatorio Chile.
}

clínicamente significativas, fueron organismos pertenecientes a la familia Bacteroidaceae.

La letalidad global de este grupo fue de un $31 \%$.

Con respecto a septicemias por microorganismos anaerobios en niños, las publicaciones son escasas.

Echeverria, Smith y colaboradores, en un periodo de 2 años (1972-1973), en el Children Hospital Medical Center de Boston, encontraron 144 hemocultivos positivos a gérmenes anaerobios, lo que correspondio a un $13 \%$ del total de hemocultivos positivos obtenidos durante ese perfodo y sólo un 9\% correspondió a pacientes con un cuadro clinico de septicemia.

En este estudio el microorganismo más frecuentemente aislado fue el Bacteroides.

En general podemos decir que los microorganismos anaerobios son habitantes normales del organismo humano, ubicándose en la cavidad buco-faríngea, intestino grueso, vagina, lugares en los cuales se encuentran en perfecto equilibrio con el resto de la flora bacteriana, siendo Bacteroides, Fusobacterium, Peptococcus, Peptostreptococcus y Clostridium los que se encuentran con mayor frecuencia.

Como microorganismos agentes de infecciones oportunistas de origen endógeno, su acción patogénica es secundaria a la nuptura de mucosas, submucosas y/o diseminación por vía sanguínea, lo que les permite invadir otros sistemas orgánicos, produciendo infecciones piogenas fulminantes.

E] desarrollo de anaerobios en otras localizaciones o "in situ" es favorecido con un potencial de óxido-reducción disminuido como consecuencia de un menor flujo sanguíneo, necrosis tisular y crecimiento de bacterias facultativas. ${ }^{1,3}$ 
En Chile no existen publicaciones sobre infecciones por anaerobios en la edad infantil. Tal situación nos motivó a investigar en forma prospectiva la incidencia y caracteres de este tipo de infecciones en el lactante.

Como grupo de trabajo nos abocamos a investigar lo que ocurte en nuestro medio en relación a los siguientes puntos:

Evaluar la respuesta a diferentes esquemas terapéuticos.

Estudiar la influencia de diversas condiciones del huésped, tales como: edad, sexo y estado nutritivo, en septicemias por gérmenes anaerobios.

Evaluar la letalidad de dichas infecciones.

\section{MATERIAL Y METODO}

Se estudiaron prospectivamente 50 pacientes hospitalizados en la Unidad de Lactantes del Hospital Roberto del Río entre mayo de 1979 y julio de 1980 , con diferentes diagnósticos y que en el momento de su ingreso y/o durante su hospitalización presentaron signos de infección grave, con gran compromiso del estado general, y que por esta razón debió practicarse hemocultivos seriados.

$\mathbf{E}_{n}$ estos pacientes se tomaron 2 muestras de sangre ( $2 \mathrm{ml} \mathrm{c} / \mathrm{u}$ ) en condiciones asépticas, usando como anticoagulante Polianetol-sulfonato de Sodio. Las muestras se mantuvieron en condiciones de anaerobiosis, para lo cual se sellaron las jeringas con tapón de goma estéril para su envío al Laboratorio de Microbiología de la Facultad de Medicina Norte, donde se procesaron en el tiempo máxímo de quince minutos.

Para la investigación de los microorganismos anaerobios se efectuó tinción de gram modificado por Hucker y simultáneamente se sembró en caldo Thyoglicolato BBL 135-C, mejorado con $\mathrm{He}$ mina y Vitamina K-1 previamente regenerado. Se incub 6 en ambiente de anaerobiosis por 48 horas (Cámara Gaspak BBL, sobre generadores de $\mathrm{CO}_{2}$ e Hidrógeno, catalizadores de Paladio, indicadores de reducción y gel de Sílice\}.
Los gérnenes en estudio se aislaron en placas de agar sangre de cordero mejorado con Vitamina K-l y Hemina y luego se procedió a la identificación de especies siguiendo la metodologia de Dowell y Howkins, del Centro de Control de Enfermedades Infecciosas de Atlanta, $y$ posteriormente se realizó su antibiograma respectivo.

Aquellos hemocultivos que fueron inicialmente negativos se observaron por un perfodo de hasta 14 dias.

Para cada muestra estudiada se llevó un protocolo en el cual se indicó: sexo, edad, estado nutricional, fecha de ingreso, diagnóstico y fecha de hemocultivos de cada paciente. En cuanto al estudio bacteriológico, se señaló el examen directo (Gram modificado por Hucker), cultivo en medios básicos, baterías de medios diferenciales y antibiograma.

Los gérmenes aerobios y facultativos aislados se identificaron siguiendo la metodologia de rutina.

\section{RESULTADOS}

El porcentaje global de hemocultivos positivos fue de un $36 \%$ ( 18 casos), de los cuales cortesponde un $12 \%$ (6 casos) a gérmenes anaerobios, $20 \%$ (10 casos) a gérmenes aerobios ylo facultabivos y un $4 \%$ ( 2 casos) a asociaciones de ambos.

\section{Tabla 1}

Distribucion de hemocultivos positivos en 50 pacientes estudiados

\begin{tabular}{lrr} 
& N. & $\%$ \\
\cline { 2 - 3 } & & \\
Hemocultivos + Gérmenes Aerobios & 10 & 20 \\
Hemocultivos + Gémenes Anaerobios & 6 & 12 \\
Hemocultivos + Asociaciones & 2 & 4 \\
Total Hemocultivos Positivos & 18 & 36 \\
\hline
\end{tabular}

De los 50 pacientes estudiados, 28 fueron lactantes del sexo masculino, 22 del sexo femenino y la distribución según hemocultivos positivos es la siguiente:

Tabla 2

Distribución por sexo de hemocultivos en 50 pacientes estudiados

\begin{tabular}{|c|c|c|c|c|c|c|c|c|}
\hline & \multirow{2}{*}{$\begin{array}{c}\text { TOTAL } \\
\text { N. }\end{array}$} & \multirow{2}{*}{$\begin{array}{c}\text { PACIENTES } \\
\%\end{array}$} & \multicolumn{2}{|c|}{ G. ANAEROBIOS } & \multicolumn{2}{|c|}{ G. AEROBIOS } & \multicolumn{2}{|c|}{ ASOCLACION } \\
\hline & & & N. ${ }^{\circ}$ & $\%$ & N. ${ }^{\circ}$ & $\%$ & N. ${ }^{0}$ & $\%$ \\
\hline Sexo masculino & 28 & 56 & 2 & 4 & 6 & 12 & 0 & 0 \\
\hline Sexo femenino & 22 & 44 & 4 & 8 & 4 & 8 & 2 & 4 \\
\hline Total & 50 & 100 & 6 & 12 & 10 & 20 & 2 & 4 \\
\hline
\end{tabular}


La edad de nuestros pacientes fluctuó entre 1 mes y 2 años, $y$ la mayor frecuencia de hemocultivos positivos la obtuvimos en pacientes de 4 y 6 meses, en que la mitad de los pacientes estudiados presentaron hemocultivos positivos tanto a gérmenes aerobios como anaerobios y/o asociación de ellos. A este grupo de enfermos pertenece además el mayor porcentaje de hemocultivos positivos a gémenes anaerobios en relación a otros grupos etarios.

Tabla 3

Distribución de hemocultivos positivos en 50 pacientes según edad

\begin{tabular}{|c|c|c|c|c|c|c|c|c|}
\hline \multirow[t]{2}{*}{ EDAD } & \multicolumn{2}{|c|}{$\begin{array}{l}\text { PACIENTES } \\
\text { ESTUDIADOS }\end{array}$} & \multicolumn{2}{|c|}{$\begin{array}{l}\text { HEMOCULTIVOS + } \\
\text { A GERMENES } \\
\text { AVAEROBIOS }\end{array}$} & \multicolumn{2}{|c|}{$\begin{array}{l}\text { HEMOCULTIVOS + } \\
\text { A GERMENES } \\
\text { AEROBIOS }\end{array}$} & \multicolumn{2}{|c|}{$\begin{array}{l}\text { HEMOCULTIVOS } \\
\text { + A ASOCIA- } \\
\text { CIONES }\end{array}$} \\
\hline & $\mathrm{N}{ }^{\circ}$ & $\%$ & N. & $\%$ & N: ${ }^{\circ}$ & $\%$ & N. ${ }^{\circ}$ & $\%$ \\
\hline l a 3 meses & 10 & 16,1 & 1 & 1,6 & 2 & 3,2 & - & - \\
\hline 4 a 6 meses & 14 & 22,5 & 3 & 4,8 & 3 & 4,8 & 1 & 1,6 \\
\hline 7 a 12 meses & 16 & 25,8 & 1 & 1,6 & 3 & 4,8 & 1 & 1,6 \\
\hline+ de 12 meses & 10 & 16,1 & I & 1,6 & 2 & 3,2 & - & - \\
\hline
\end{tabular}

Con respecto al estado nutricional de nuestros pacientes, 22 fueron eutroficos, 14 desnutridos grado I (peso, talla), 7 desnutridos grado II y 7 desnutridos grado III.

Tabla 4

$\begin{array}{lllll}\text { Eutróficos } & \text { D.1 } & \text { D.2 } & \text { D.3 } & \text { Total }\end{array}$
N. ${ }^{\circ}$ Paciente
22
14
7
7
50

De los 18 pacientes con hemocultivos positivos, 10 correspondieron a niños cuya relación pesotalla fue nonmal y 8 presentaban alteraciones nu- tricionales en diversos grados, la que se detalla en la Tabla 5.

Tabla 5

Distribución de pacientes con hemocultivos positivos según estado nutricional

\begin{tabular}{lcccc}
\hline & G. ANAEROBIOS & G. AEROBIOS & $\begin{array}{c}\text { ASOCIACIONES } \\
\text { AERO-ANAEROBIO }\end{array}$ & $\begin{array}{c}\text { TOTAL } \\
\text { PACIENTE }\end{array}$ \\
\hline Eutróficos & 4 & 5 & 1 & 10 \\
D.1 & 1 & 2 & - & 3 \\
D.2 & -1 & 2 & -1 & 2 \\
D.3 & 1 & 1 & 1 & 3 \\
\cline { 2 - 5 } \multicolumn{1}{c}{ Total } & 6 & 10 & 2 & 18 \\
\hline
\end{tabular}

En relación a la distribución etiológica de los 6 pacientes en que se aislaron gérmenes anaerobios podemos señalar que el Bacteroides fragilis y el Propionibacterium acné fueron los microorganismos más frecuentes, como se puede observar en la Tabla 6. 
Tabla 6

Distribución de gérmenes anaerobios en 6 pacientes con hemocultivos +

\begin{tabular}{|c|c|}
\hline ESPECIE & N. ${ }^{\circ}$ DE PACIENTES \\
\hline Bacteroides fragilis & 2 \\
\hline Propionibacterium acné & 2 \\
\hline Clostridium perfringens & $\mathbf{l}$ \\
\hline Peptococcus sacharolyticus & 1 \\
\hline Total & 6 \\
\hline
\end{tabular}

En los 10 pacientes con Hemocultivos positivos a gérmenes aerobios los microorganismos aislados en mayor frecuencia fueron Staphylococcus aureus y Staphylococcus epidermidis.

Los otros gérmenes encontrados se distribuyeron entre diferentes especies de Bacilo Gram (-), como veremos a continuación en la Tabla 7 .
Tabla 7

Distribución de gérmenes aerobios en 10 pacientes con hemocultivos +

\section{ESPECIE}

PACIENTES

\begin{tabular}{lc} 
Staphylocuecus epidemidis & 3 \\
Staphylococeus aureus & 2 \\
Klebsiella pneumoniae & 1 \\
Salmonella para-typhi B & 1 \\
Aeromona tidrophyla & 1 \\
Proteus vulgaris & 1 \\
Bacilos Gram (-) No Fermentador & 1 \\
\cline { 2 - 2 } \multicolumn{1}{c}{ Total } & 10 \\
\hline
\end{tabular}

De los hemocultivos estudiados en 2 pacientes obturvimos asociación de gérmenes Aerobios y Anaerobios.

Tabla 8

Pacientes con asociación de gérmenes aerobios y anaecobios

\begin{tabular}{|c|c|c|}
\hline & HEMOCULTIVON. ${ }^{\circ} 1$ & HEMOCULTIVO N. 2 \\
\hline $\begin{array}{l}\text { Pacientes N. }{ }^{\circ} 1 \\
\text { Pacientes N. }{ }^{\circ} 2\end{array}$ & $\begin{array}{l}\text { Peptococeus sacharolyticus } \\
\qquad(-)\end{array}$ & $\begin{array}{l}\text { Klebsiella pneumoniae } \\
\text { Staphylococcus epidermidis } \\
\text { Peptucnecus sacharolyticus }\end{array}$ \\
\hline
\end{tabular}

Síndrome diarreico agudo y bronconeumonta bilateral fueron los diagnósticos de ingreso más frecuentes en nuestros pacientes con hemoculti- vos positivos (8 y 6 casos, respectivamente), el resto se distribuye en los diagnósticos de Síndrome Febril, Vasculopatia y Leucemia.

\section{Tabla 9}

Diagnóstico de ingreso de los pacientes con hemocultivos positivos

\begin{tabular}{lccc}
\hline $\begin{array}{c}\text { DIAGNOSTICO DE } \\
\text { INGRESO }\end{array}$ & $\begin{array}{c}\text { MICROORGANISMOS } \\
\text { AEROBIOS }\end{array}$ & $\begin{array}{c}\text { MICROORGANISMOS } \\
\text { ANAEROBIOS }\end{array}$ & $\begin{array}{c}\text { ASOCIACIONES } \\
\text { AERO-ANAEROBIOS }\end{array}$ \\
\hline B.R.N. & 5 & 1 & - \\
S.D.A. & 3 & 3 & 2 \\
S. Febril & 1 & 1 & 0 \\
Vasculopatia \\
Leucemia
\end{tabular}


Tabla 10

Pacientes con hemocultivos positivos y cradro clínico compatible

\begin{tabular}{lcccccc}
\hline & \multicolumn{2}{c}{ G. ANAEROBIOS } & \multicolumn{2}{c}{ G. AEROBIOS } & \multicolumn{2}{c}{ ASOCIACIONES } \\
& N. ${ }^{\circ}$ & $\%$ & N. & \% & N. \\
\hline $\begin{array}{l}\text { Hemocultivos positivos } \\
\begin{array}{l}\text { Hemocultivos positivos } y \\
\text { cuadro clínico compatible }\end{array}\end{array}$ & 6 & 100 & 10 & 100 & 2 & 100 \\
\hline
\end{tabular}

De todos los pacientes en que se aislaron gérmenes anaerobios con o sin asociación, sólo en tres de ellos el cuadro clínico que presentaron fue compatible con una septicemia (sindrome febril, hepatomegalia, compromiso del estado general), aislándose Bacteroides fragilis, Clostridium perfringens y la asociación de Peptococcus sacharolyticus y Klebsiella pneumoniae en 2 hemoxultivos, respectivamente.

Las características clínicas más conocidas de estos pacientes se resumen en el cuadro siguiente.

\section{Tabla 11}

Signos c'línicos más frecuentes concomitantes al hallazgo de hemocultivos positivos a génnenes anaerobios y/o asociación

- Deposiciones líquidas

- Deshidratación

- Signos de coagulación intravascular diseminada (Petequiąs, Sangramiento en Sitio de Punción)

- Fiebre

Con el objeto de conocer la sensibilidad de los microorganismos a los diferentes antimicrobianos se practicó antibiograma por difusión en agar según método de Kirby y Bauer modificado.

Tabla 12

\begin{tabular}{lcccccc}
\hline & PENICILINA & CAF & GENTAMICINA & LINCO & CLINDA & ERITRO \\
\hline $\begin{array}{l}\text { Bacteroides } \\
\text { fragilis (2) }\end{array}$ & R & S & R & S & S & R \\
$\begin{array}{l}\text { Propionibacterium } \\
\text { acné }(2)\end{array}$ & S & S & R & S & S & S \\
$\begin{array}{l}\text { Peptococius } \\
\text { sacharolyticus (1) }\end{array}$ & S & S & R & S & S & S \\
$\begin{array}{c}\text { Clostridium } \\
\text { perfringens (1) }\end{array}$ & S & S & S & S & S & S \\
\hline
\end{tabular}

\section{COMENTARIOS}

De Ios 50 pacientes estudiados, en 18 de ellos obtuvimos hemocultivos positivos ( $30 \%$ ). Aislamos microorganismos anaerobios en 6 pacientes $y$ de éstos sólo en 3 la evolución del cuadro clínico es compatible con una septicemia, correspondiendo éstos a Bacteroides fragilis, Clostridium perfringens y Peptococcus sacharolyticus asociado con Klebsiella pneumoniae.
El resto de gérmenes anaerobios aislados (3 casos) correspondió a bacteremias transitorias y/o a contaminación de las muestras. Tal situación aparentemente corresponde al aislamiento de Propionibacterium acné (2 pacientes), germen comensal de la piel, y a Bacteroides fragilis, que dio lugar a una bacteremia transitoria debido a que se comprobó una infección del Tracto Urinario posteriormente.

El porcentaje de positividad en los hemoculti- 
vos es más alto que el que se encuentra en la mayoria de las publicaciones, lo que probablemente se pueda explicar por tratarse de un universo seleccionado.

La letalidad de los pacientes con hemocultivos positivos a gérmenes anaerobios fue de $100 \%$, lo cual podría estar indicando la gravedad y pronóstico de dichas infecciones.

Con respecto a los gérmenes aerobios aislados, la especie más frecuente fiue Staphylococcus aureus, seguido por Klebsiella pneumoniae.

Síndrome diarreico agudo y bronconeumonía bilateral fueron los diagnósticos de ingreso más frecuentes en nuestros pacientes; esto guarda relación con la patología más frecuente que motiva el ingreso en una unidad de lactantes.

En relación a la distribución por sexo, estado nutritivo y edad de los pacientes afectados, no se observan diferencias significativas en la incidencia de septicemias de gérmenes aerobios, anaerobios y/o asociaciones.

El cuadro clínico, en aquellos pacientes en que se aislaron microorganismos anaerobios no difiere fundamentalmente en sus características de los procesos septicémicos provocados por microorganismos aerobios Gram negativos, destacando la presencia de deposiciones líquidas, con deshidratación persistente y signos clínicos y de laboratorios de coagulación intravascular diseminada.

Es interesante observar que ninguno de los casos demostró compromiso meningoencefálico evidenciado por la negatividad persistente de anormalidad en el L.C.R.

En la literatura extranjera la frecuencia de asociaciones de gérmenes aerobios y anaerobios es de alrededor del $60 \%$, muy diferente a nuestra casuística, que fue notoriamente menor $(2 \%)$. Esto podria explicarse por ser grupos no comparables en edad y patología.

Con respecto a la sensibilidad a los antimicrobianos, todos los gérmenes anaerobios aislados, con excepción del $B$. fragilis, fueron senșibles a penicilina cloramfenicol, lincomicina, clindamicina $y$ eritromicina.

El Bacteroides present 6 resistencia a penicilina, gentamicina y eritromicina y sensibilidad a cloramfenicol, lincomicina y clindamicina.

No fue posible evaluar la respuesta a diferentes esquemas terapéuticos en los pacientes con septicemia por gémenes anaerobios, por el curso rápidamente mortal de ésta.

La posibilidad de que la presencia de gérmenes anaerobios sea la responsable de cuadros graves del lactante, nos obliga a investigar dichos gérmenes como un método de rutina.

\section{RESUMEN}

Se estudiaron prospectivamente 50 pacientes hospitalizados en la Unidad de Lactantes del Hospital Roberto del Río, entre mayo de 1979 y julio de 1980, con diferentes diagnósticos y que en el momento de su ingreso y/o durante su hospitalización presentaron signos de infección grave y que por esta razón se practicaron hemocultivos seriados en búsqueda de gérmenes aerubios y anaerobios.

Aislamos microorganismos anaerobios en 6 pacientes y de éstos, sólo en 3 la evolución del cuadro clínico es compatible con una septicemia. Los agentes etiológicos encontrados fueron Bacterojdes fragilis, Clostridium perfringens y Peptococcus sacharolyticus, este último asociado a KlebsieIla pneumoniae.

Esta experiencia ilustra la importancia de investigar la presencia de anaerobios en todo cuadro infeccioso grave del lactante.

\section{REFERENCIAS}

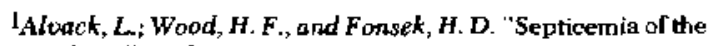
newborn". Pediatric. Clin. North An. 13: 1131, 1966.

${ }^{2}$ Ditnkle, L. M.; Brotherton, T, J., and Feigin, A. D. "Anaerobic Infections in Children: A prospectic study". Pediatrics 5: $311,1976$.

${ }^{3}$ Echeverria, P.; M.D., Amold, L.; Smith, M.D. Anaerobic Bacteremia as observed in a Children's Hospital", Clinical Pediatrics September 1978. Vol. 17, N. ${ }^{\circ} 9$ (689-695).

4Finegold, J.; Bartlett, A. W.; Choow, D. J.; Flora, S. L.; Gorbach, E. /.; Harder and F. D. "Management of Anaerobic Infections", S. M. Tally, LCLA Conference. Annals of Intemal Medicine, Vol. 83, N. ${ }^{\circ} 3$, September 1975.

5Gorbach, S. I.; Eartiett, J. G. "Anaerobic Infections". New England J. Med. 290: $1177,1184,1237-1245,1249-1294,1974$.

6Pearson, H. E., and Anderson, C. V. "Perinatal deaths associated with Bactervides infections". Obstet. Gynecol. 30: $486,1967$.

7Valenzuela, M. E.; Guertero, B.; Sanhueza, A. "Sensibilidad de Anaerobios en Infecciones Ginecoobstétricas". Trabajo en publicación.

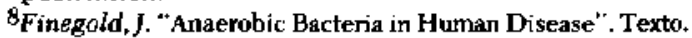

9Wilecr, R.; Wolter; Martin, W, et al. "Anaerobic Bacteremin". Mavo Clin. Proc, Sept 1972, Vol 47.

10 Marhios, R. C.; Harding, G. K. M.; Grintwith, M. J. "Bacteremia Due to Bacteroidaceae. A review of 92 cases". The Joumal of Infections Diseases. Vol. 135. Supplement. March 1977.

${ }^{1}$ Ballows, A.; Dehaan, R,; Dotoell, V. R.; Guze, L. B. "Anaenobic bacteria". USA, Illinois. Springtield; Edit Charles C. Thomas, 1974.

I2Helge Otsen. "Bacteroides Bacteremia". Scand. Infect. Dis. 8; $107-11,1976$.

13 Awat, J. A.; Valenzuela, M. E.; Bustos, R. "Investigación de 
bacterias anaerobias en otikis media supurada". Rev. Otorrinolaringologica 37: 56-60, 1977.

${ }^{14}$ Laurense, P. F,; Tietjen, G. W.; Gungrich, S.; King, T. C. "Bacteroides Bacteremia", Ann. Surg. November 1977.

15 Pearson, T, Braine, H.; Fathbun, H. "Corynehacterium sepsis in Oncology Patients". JAMA, Oct 17, 1977. Vol. 238, N. ${ }^{\circ} 16$.

lbPong, G. L; Cirile, E. A. "Fusobacterium septicemia with otitis media and mastoiditis". Postgraduate Medicine, Vol. 57 . N. ${ }^{\circ} 54$, April 1975.

17 Finegold, S. M. "Antibiotic Suscepribility patterns as gids in classification and characterization of Gram negative anoerobic bacilli". J. Bact. 94: 1443, 1967.

18 Hobinow, M.; Simonelti, F.A. "Fusobacterium bacteremia in the Newborn". Ann. J. Dis. Child. 110: 92, 1965.

${ }^{19}$ Chaw, A. W.; Teake, R. D.; Yamanchi, T, et al. "Significance of anaerobic in Neonatal Bacteremia". Pediatrics 54: 736, 1974.

${ }^{20}$ Sanders, $D . V$, and Stevenson, $J$. "Bacteroides infections in children". J. Pediatric 72; 673, 1968.

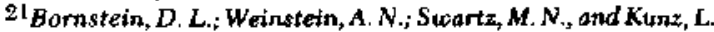
S. "Anaerobic infections: A review of current experience". Medícine 43: 207, 1964.

22. Burton, H. C.; Kletn, S. W., and Lane, R. B. "Septicemia in premature infants". Am. J. Dis. Child. 1 10: 29, 1965.

${ }^{23}$ Horrod, J. R., and Stevens, $D, A$. "Anaerobic infections in the newborn infant". J. Pediatric 85: 399, 1974 